\title{
A LINGUOVERTED IMPACTED TOOTH WITH OROCUTANEOUS FISTULA - A RARE CASE REPORT
}

\author{
SRIKANTH $\mathbf{G}^{\mathbf{1}}$, ABHAY T. KAMATH ${ }^{\mathbf{1}}$, ADARSH KUDVA ${ }^{\mathbf{1}}$, ANUPAM SINGH ${ }^{1}$, \\ KOMAL SMRITI ${ }^{2}$, SUNITHA CARNELIO ${ }^{3}$
}

\author{
${ }^{1}$ Dept. of Oral \& Maxillofacial Surgery, MDS, Manipal College of Dental Sciences, \\ Manipal Academy of Higher Education, Manipal, India \\ ${ }^{2}$ Dept. of Oral Medicine \& Radiology, MDS, Manipal College of Dental Sciences, \\ Manipal Academy of Higher Education, Manipal, India \\ ${ }^{3}$ Dept. of Oral Pathology, MDS, Manipal College of Dental Sciences, Manipal \\ Academy of Higher Education, Manipal, India
}

\begin{abstract}
The orocutaneous fistulous tract of odontogenic origin is often a diagnostic challenge, due to its rare manifestation and absence of dental signs and symptoms. The odontogenic cutaneous fistula is often misdiagnosed as a superficial skin lesion of non-odontogenic origin delaying the treatment. The diagnosis and treatment must be precise and swift to improve the clinical outcome and minimize the complications.

This article presents a rare case of odontogenic keratocyst involving a linguoverted impacted third molar presenting as orocutaneous fistula. The patient was initially treated with empirical antibiotic therapy with no resolution of the cutaneous fistula and thickening of the skin around the sinus opening resulting in cosmetic deformity. Once the fistula was attributed to the underlying odontogenic cyst, treatment was done by cyst enucleation along with the extraction of tooth and fistula excision. The purpose of the paper is to emphasize the importance of early and accurate diagnosis and prompt management of the orocutaneous fistula due to the odontogenic origin.
\end{abstract}

Keywords: orocutaneous fistula, linguoversion, odontogenic keratocyst

\section{Introduction}

A fistula is defined as a pathological communication between two anatomic spaces or a trail connecting an internal cavity to the body surface, whereas a channel starting or ending into one opening is termed as a sinus tract. Both the terminologies are often used interchangeably in the literature. An odontogenic cutaneous fistula can be defined as an abnormal pathway between the oral cavity and the skin surface of the maxillofacial region. A fistula of odontogenic origin is an unusual entity but well known in the medical and dental literature [1,2]. In the beginning the lesion is usually considered to be of dermatological origin, and thus the patient approaches a physician or a skin specialist. Following which starts the long-term multiple antibiotic therapies along with biopsies, if the lesion fails

Manuscript received: 03.12.2017

Received in revised form: 21.01.2018

Accepted: 30.01.2018

Address for correspondence: komalmds1@gmail.com to heal. Delay and incorrect diagnosis make the disease chronic in nature leading to cosmetic comorbidity.

Cutaneous fistula of dental origin usually develops as a sequel to carious lesion or trauma causing a breach in enamel, dentine and ultimately involving pulp tissues [3]. If treatment is not done at this stage, the pulp tissues become necrotic, and infection extends beyond the apex of the tooth into the periapical area leading to a periapical abscess that eventually traverses the track of least resistance and breaks out through the skin surface [4]. Apart from pulp pathology, pericoronal infections and malignancy can also cause an orocutaneous fistula, but it is rare. Often the pericoronal area endures partially erupted or impacted third molar around which various pathologies can flourish. Inflammatory changes and cyst formation have been reported in the pericoronal region of even completely impacted asymptomatic third molars [5]. Literature search accounts for the development of numerous odontogenic cysts and odontogenic tumor-like ameloblastoma around 
impacted third molars suggesting the pathologic potential of pericoronal tissues. Hence pericoronal infection can be the first indication of the existence of such pathology. If such lesions are left untreated for a long time, they can manifest as cutaneous fistulas. These pathologies in their initial stages often go unnoticed, if they are asymptomatic and pose a diagnostic challenge. Here we present a rare case of orocutaneous fistula associated with odontogenic keratocyst involving linguoverted impacted lower left third molar.

\section{Case Report}

A 23-year-old otherwise healthy male was referred from the department of dermatology to the outpatient department of Oral and Maxillofacial Surgery, with the chief complaint of a draining lesion from the left side of the face for the past three months. On taking the detailed history of the presenting illness, the patient reported that he first experienced pain in the lower left cheek region five months ago, which was mild, dull aching in nature. Two days later mild swelling also appeared in the same area. He consulted a general physician who prescribed him antibiotics and analgesics which brought symptomatic relief but pain and swelling re-appeared after seven weeks. However, this time he also noticed an opening on the left side of the face with foul-smelling yellowish discharge. $\mathrm{He}$ was referred to a dermatologist for further management. He was treated with various topical and systemic antibiotic for two months based on the diagnosis of superficial skin infection. Later he was referred to us to rule out the probability of odontogenic cause for the lesion as it was not healing. His medical history was non-contributory, and there was no previous history of trauma to that area. The patient never had tooth pain and did not consult any dentist regarding this problem. However, he mentioned a difficulty in chewing from the left side. On extraoral examination, an erythematous area measuring $0.5 \mathrm{~cm} x$ $1 \mathrm{~cm}$ on left lower $1 / 3 \mathrm{rd}$ of the face with an opening in the center with intermittent discharge was found (Figure 1). The surrounding area appeared retracted and crusted. On palpation, the region was firm in texture, and scanty purulent exudate came out from the opening on applying digital pressure. The discharge swab was sent for the culture which was sterile with no growth of microorganisms. A 28 gauge stainless steel wire was inserted through the opening to trace the path and cause of the lesion. The wire got embedded entirely through the sinus but failed to connect to the oral cavity posing a diagnostic dilemma. Intra-oral examination revealed mild erythema associated with oral mucosa surrounding the intact left lower second molar and impacted third molar (Figure 2). No obliteration of vestibule and no pus discharge was noticed intra-orally. On palpation, there was mild tenderness with thinning of buccal cortex in the region of an impacted third molar. The patient did not complain of paresthesia. None of the teeth in the oral cavity were carious. Hence, correlating history and clinical examination a clinical diagnosis of orocutaneous fistula due to pericoronal infection around impacted third molar was formulated.

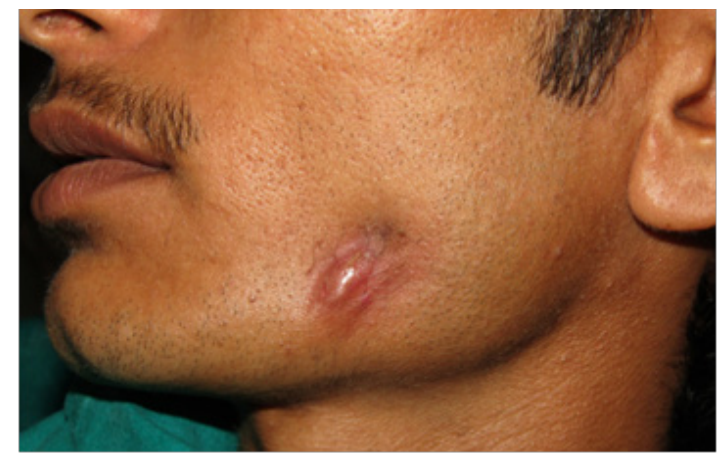

Figure 1. Preoperative photograph showing erythematous area measuring $0.5 \mathrm{~cm} \times 1 \mathrm{~cm}$ on left lower $1 / 3^{\text {rd }}$ of the face with an opening in the center.

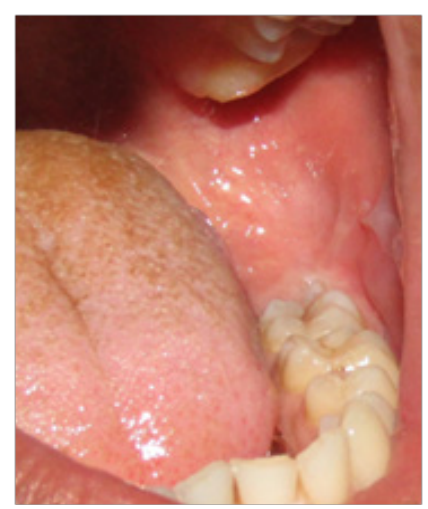

Figure 2. Intra-oral view revealing mild erythema of intact oral mucosa surrounding left lower $2^{\text {nd }}$ molar and impacted $3^{\text {rd }}$ molar.

A panoramic screening radiograph was taken after passing gutta percha through the fistula to confirm the odontogenic cause for its formation which revealed a large solitary well-defined radiolucency involving the lower left impacted third molar which was placed buccolingually placed (Figure 3). As the complete assessment of the lesion was difficult with a conventional radiograph, Cone Beam Computed Tomography (CBCT) was done for three-dimensional evaluation of the impacted 3rd molar, associated cystic cavity and its relation with inferior alveolar nerve canal. Detailed analysis illustrated that there was a large single well-defined radiolucency with a sclerotic border around impacted third molar in the third quadrant extending from distal to the second molar up to anterior part of ascending ramus, measuring $3 \mathrm{~cm} \mathrm{x} 4 \mathrm{~cm}$ x $2.5 \mathrm{~cm}$ in dimension. The radiolucent lesion was found to be in close approximation with the superior border of inferior nerve canal. Furthermore, the impacted 3rd molar was in linguoversion, with the root apex facing the buccal cortex and crown embedded in the lingual cortical plate. 
Sagittal sections of CBCT near the root apex showed cortical thinning with a perforation which would have resulted in infection of the cystic lesion which drained as orocutaneous fistula. Hence, correlating the clinical and radiographic features, oro-cutaneous fistula due to infected Dentigerous cyst was formulated as a provisional diagnosis. Infected odontogenic keratocyst (OKC) and unicystic ameloblastoma were considered as differential diagnosis. An incisional biopsy was done, and the content was sent for histopathological examination. The microscopic examination revealed a cystic lumen associated with 38 lined by a para keratinized stratified epithelium of 4-6 cell layer thickness with a corrugated surface and the lesion was confirmed as odontogenic keratocyst (Figure 4).

Under all aseptic precautions, the patient underwent surgical removal of the impacted 3rd molar along with cyst enucleation and excision of the oro-cutaneous fistula under

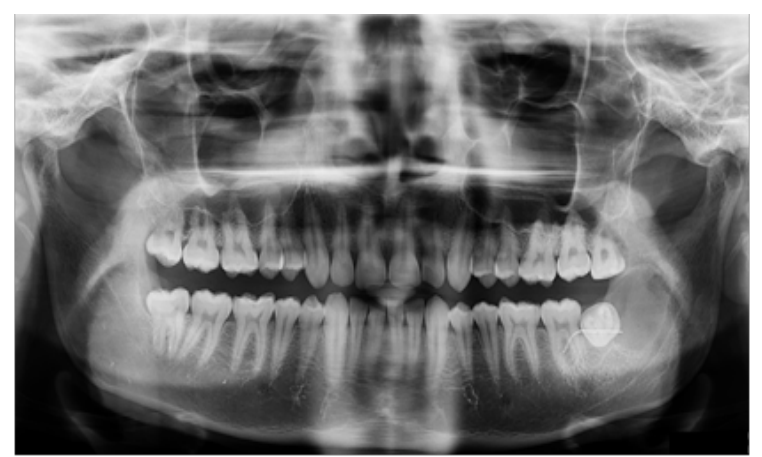

Figure 3. Panoramic radiograph showing solitary radiolucency distal to impacted left lower third molar. The impacted third molar in horizontal orientation and Guttapercha tracing from extra-oral opening leading to the radiolucency suggesting odontogenic origin. general anesthesia. Standard modified ward's incision was placed intraorally and linguoversion impacted 3rd molar was removed at first by sectioning method (Figure 5). The cystic enucleation was performed, followed by curettage (Figure 6). Then the fistulous tract was approached from the extraoral site. An elliptical incision was made around the fistulous tract and surrounding erythematous region. The fistula was dissected from the surrounding tissues and traced intraorally till the lateral border of the mandible. The extra-oral opening of the fistula with the skin component was rotated intra-orally and excised in-toto (Figure 7). Then chemical cauterization of the enucleated cystic cavity was done with modified Carnoy's solution. Carnoy's solution was applied for 3-4 minutes followed by thorough irrigation. Fine extraoral sutures were placed to enhance esthetic healing with minimal comorbidity. At six months follow-up, the patient showed complete resolution of the fistulous tract (Figure 8).

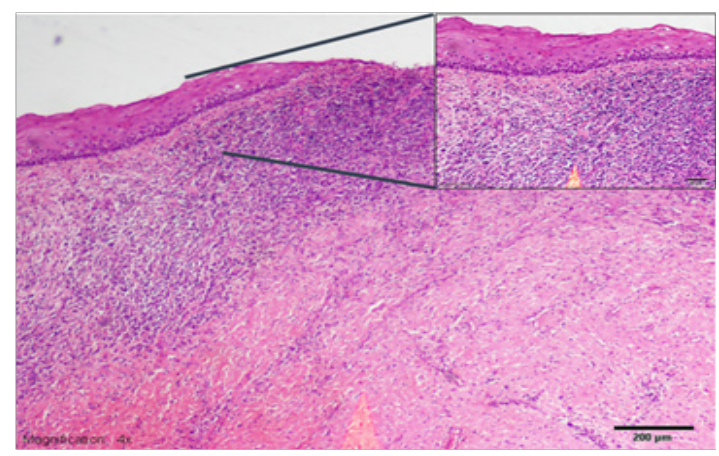

Figure 4. Pictomicrograph of the tissue taken during incisional biopsy. (Inset) On higher magnification - para keratinized stratified epithelium of 4-6 cell layer thickness. The basal cells were tall, columnar with a hyperchromatic palisading nucleus and the capsular tissue comprised of dense bundles of collagen, fibroblasts, fibrocytes and blood vessels with dense infiltration of chronic inflammatory cells suggestive of Odontogenic keratocyst.

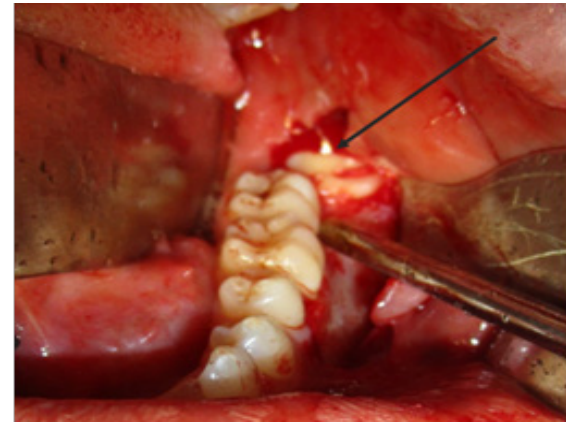

Figure 5. Intra-operative view showing impacted left lower third molar in linguoversion.

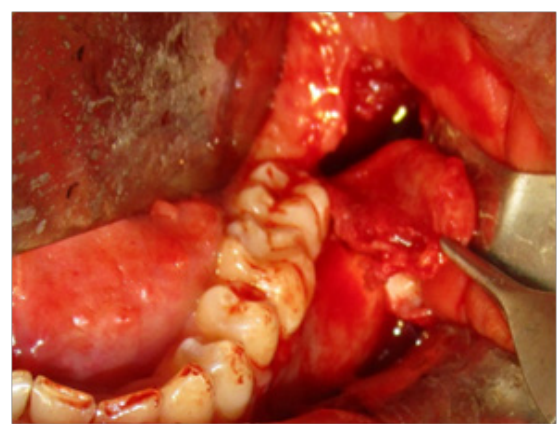

Figure 6. Cystic lining being enucleated after removal of the impacted third molar. 


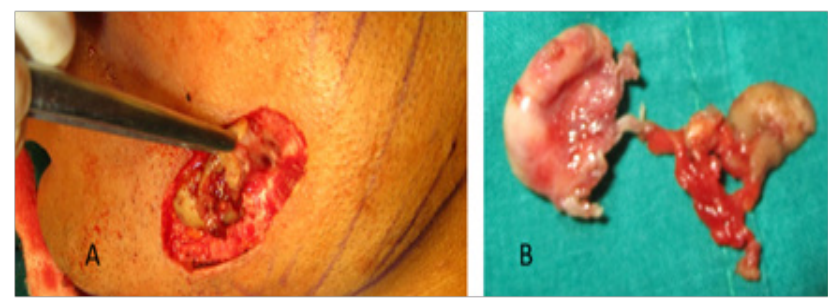

Figure 7. (A) Extra-oral elliptical skin incision around the fistula; (B) Excised Fistula tract with the cystic lining.

\section{Discussion}

A fistula is an abnormal pathway connecting two epithelized surface. As the name is self-explanatory, an orocutaneous fistula is an abnormal communication between oral cavity and skin surface. There are various causes of draining sinuses on the face which include chronic osteomyelitis, fistula due to salivary gland disorder, congenital fistula, deep mycotic infection, and malignancy. However, skin lesions such as pustules, furuncles, foreign body lesions may have similar appearance superficially as an orocutaneous tract, but they are not the real sinus tracts [6]. The probable cause of occurrence of such entity is undiagnosed or improperly treated pulpal pathology. Most frequent reason for the existence of such lesion is the diagnostic delay which results in chronicity of the underlying infection hence leading to the expulsion of the underlying pathology as fistula [7].

Also, an impacted tooth may harbor bacteria and result in pericoronitis. Based on the inclination of third molar with respect to second molar, an impacted tooth can be categorized as vertical, horizontal, mesioangular or distoangular. However, two more rare forms of impactions are also reported which is called buccoversion and linguoversion impaction in which crown of the tooth faces buccal cortical plate and root lies towards the lingual cortical plate and vice versa. Infection around such tooth leads to cortical thinning and may drain as orocutaneous fistula. The origin of odontogenic cyst in association with impacted third molar is a common finding. The most common being Dentigerous cysts followed by uni-cystic ameloblastoma and odontogenic keratocyst. They may arise from the homogeneous radiolucent dental follicle surrounding the unerupted tooth or its remnants.

Odontogenic keratocyst may be found in association with an impacted 3rd molar or may form independently. However, the study conducted by Tsukamoto et al. states that the $\mathrm{OKC}$ associated with an impacted tooth illustrates aggressive form than its counterpart [8]. It is often challenging to clinically differentiate an odontogenic keratocyst from any other cystic lesion of the jaw. Due to similarity in both clinical and radiographic appearance, odontogenic keratocyst remains a diagnostic challenge

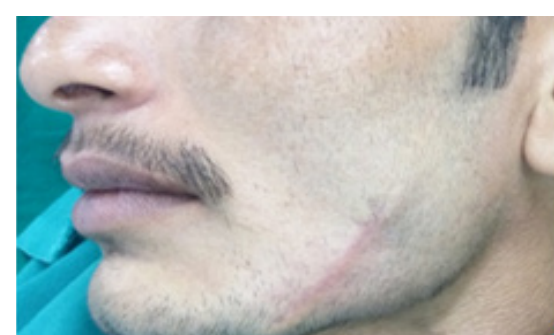

Figure 8. Follow-up after 6 months showing complete resolution of the fistula with minimal scarring. for the clinicians. As the keratocyst tend to expand in the medullar cavity rather than producing a cortical expansion; a clinically visible expansion occurs very late in the course of the disease. Hence, patients are asymptomatic until cyst reaches a large size, and in some case might even cause pathologic fractures [9]. However, OKC causing draining fistula as present in this case is uncommon and there is paucity of literature as only 1 case has been reported by Zacharaides et al., which shows impacted tooth in association with $\mathrm{OKC}$ with extra-oral draining sinus [10]. No case has been reported in the literature about $\mathrm{OKC}$ associated with linguoverted tooth with extra-oral fistula. Due to the high propensity of pericoronitis in the mandibular third molar region, it is not uncommon for the underlying cyst to develop superadded infection. The low oxygen tension along with increased serum transudate in the area facilitates proliferation of pathogenic bacteria and result in destructive activity [11]. The bacteria, which are predominantly anaerobic produce a variety of proteolytic enzymes, exotoxins, and endotoxins which lead to a significant amount of tissue destruction and lysis [12]. This process results in the formation of an abscess cavity with suppuration. If left untreated, over a period of time, it progresses to form a fistulous tract through alveolar bone involving surrounding soft tissue and creating an opening extra-orally. Hence a detail history and thorough clinical examination can give a clue to appropriate diagnosis. Unerupted third molars should be viewed with suspicion. Orthopantomogram as screening radiograph of the jaw should be advised to evaluate the third molar status in young individuals. Some authors have even suggested prophylactic removal of impacted third molars due to the high incidence of pathologies around them [13].

CBCT imaging is of great importance to evaluate the true extent of $\mathrm{OKC}$ three-dimensionally. As the proliferative characteristics of the cystic epithelium and connective tissue result in multicentric simultaneous growth, it leads to the formation of satellite cysts and epithelial islands in the connective tissue, which explains the high relative recurrence rates of $\mathrm{OKC}$. Hence, the inability to accurately assess the distance from the central cyst at which these 'satellite-cysts' can be seen in the alveolar bone, have led to 
various opinions for management of OKC [14]. Apart from that patient's individual features like, his general health status, age, and reliability for follow-up are some of the critical determinants that regulate the treatment outcome. Essential characteristics of the lesion which govern the treatment outcome include: whether the cyst is solitary, multiple, or recurrent; radiographic borders, presence or absence of tooth within the lesion and cyst location [15]. Owing to young age of the patient and relatively smaller size of the intra-bony cyst, patient was managed by cyst enucleation and chemical cauterization. Various other treatment modalities have been suggested to reduce the recurrence rate, like curettage, peripheral osteotomy, osseous resection in the form of marginal or segmental osteotomies, removal of mucosa in cases of cortical perforation. Access limitation and incomplete removal of the $\mathrm{OKC}$, owing to the size and location of the cyst, is often associated with increased comorbidity and esthetic compromise.

A multidisciplinary approach is essential to resolve the diagnostic dilemma as not all the lesions occur in the proximity of odontogenic infection hence patient may end up consulting a skin specialist or general physician. Odontogenic infections may often go unnoticed if pain or swelling is absent. Accordingly, a dental opinion must be obtained to achieve correct diagnosis in the lesions associated with the maxillofacial region and appropriate referral should be made for proper management.

\section{Conclusion}

An orocutaneous fistula may find its origin from odontogenic as well as non-odontogenic pathology. The odontogenic cutaneous fistula is uncommon but well documented. Such lesions can be mistaken as cutaneous lesion hence a detailed history with appropriate investigation and supportive referrals between physicians, a dermatologist, is required along with dental opinion to obtain the correct diagnosis and precise treatment. Early diagnosis of the lesion helps in the treatment of the patient with minimal distress and esthetic complications, minimizing the chances of added complications. Because of the unpredictable and aggressive nature of the cyst, various presentations of an $\mathrm{OKC}$ have been reported in the literature. However, an $\mathrm{OKC}$ with a superadded pericoronitis infection and resulting in an oro-cutaneous fistula is a unique presentation. Nevertheless, it still demands to be managed aggressively, which in this case was done by combination therapy of enucleation of the cyst with chemical cauterization along with excision of the fistulous tract and the associated mucosa.

\section{References}

1. Yasui H, Yamaguchi M, Ichimiya M, Yoshikawa Y, Hamamoto Y, Muto M. A case of cutaneous odontogenic sinus. J Dermatol. 2005;32(10):852-855.

2. Mittal N, Gupta P. Management of extra oral sinus cases: a clinical dilemma. J Endod. 2004;30(7):541-547.

3. Cantatore JL, Klein PA, Lieblich LM. Cutaneous dental sinus tract, a common misdiagnosis: a case report and review of the literature. Cutis. 2002;70(5):264-267.

4. Cheung A, Hudson JW, Viehweg T. Unique pericoronal orocutaneous fistula: sequelae of chronic subclinical inflammation. J Oral Maxillofac Surg. 2005;63(11):1676-1679.

5. Simşek-Kaya G, Özbek E, Kalkan Y, Yapici G, Dayi E, Demirci T. Soft tissue pathosis associated with asymptomatic impacted lower third molars. Med Oral Patol Oral Cir Bucal. 2011;16(7):e929-e936.

6. Gupta M, Das D, Kapur R, Sibal N. A clinical predicamentdiagnosis and differential diagnosis of cutaneous facial sinus tracts of dental origin: a series of case reports. Oral Surg Oral Med Oral Pathol Oral Radiol Endod. 2011;112(6):e132-e136.

7. Patni PM, Jain P, Hiremath H, Raghuwanshi S, Vijaywargia P, Patni MJ. Cutaneous dental sinus of a submental region: an eight years follow-up. Clujul Med. 2017 Jul 25; doi:10.15386/cjmed-812. 8. Tsukamoto G, Makino T, Kikuchi T, Kishimoto K, Nishiyama A, Sasaki A, et al. A comparative study of odontogenic keratocysts associated with and not associated with an impacted mandibular third molar. Oral Surg Oral Med Oral Pathol Oral Radiol Endod. 2002;94(2):272-275.

9. Shear M. Odontogenic keratocysts: clinical features. Oral Maxillofac Surg Clin North Am. 2003;15(3):335-345.

10. Zachariades N, Papanicolaou S, Triantafyllou D. Odontogenic keratocysts: the review of the literature and report of sixteen cases. J Oral Maxillofac Surg. 1985;43(3):177-182.

11. White RP Jr, Madianos PN, Offenbacher S, Phillips C, Blakey GH, Haug RH, et al. Microbial complexes detected in the second/ third molar region in patients with asymptomatic third molars. J Oral Maxillofac Surg. 2002;60(11):1234-1240.

12. Peterson L. Principles of surgical and antimicrobial infection management. In: Topazian RG, Goldberg MH, Hupp JR (eds), Oral and Maxillofacial Infections (ed 4). Philadelphia, PA, WB Saunders, 2002, p 103.

13. Ahlqwist M, Gröndahl HG. Prevalence of impacted teeth and associated pathology in middle-aged and older Swedish women. Community Dent Oral Epidemiol. 1991;19(2):116-119.

14. Ghali GE, Connor MS. Surgical management of the odontogenic keratocyst. Oral Maxillofac Surg Clin North Am. 2003;15(3):383-392.

15. Pavelić B, Katunarić M, Segović S, Karađole MC, Katanec $\mathrm{D}$, Saban A, et al. The incidence of satellite cysts in keratocystic odontogenic tumors. Coll Antropol. 2014;38(1):269-273. 